\section{Leitbild Geographie Schweiz}

\section{Einführung}

Raum- und Umweltprobleme beschäftigen unsere Gesellschaft mehr denn je. Seit ihren Anfängen befaßt sich die Geographie mit diesen Fragen. Es ist daher wichtig, daß wir unsere Fragestellungen bekanntmachen und die Voraussetzungen schaffen, die Ziele unserer Disziplin besser verwirklichen zu können. Ein einzigartiges Merkmal der Geographie ist, daß sie sowohl in den Natur- als auch in den Sozial-, Wirtschafts- und Geisteswissenschaften verankert ist und Beziehungen zu Ingenieur- und Rechtswissenschaft pflegt. Diese thematische Breite eröffnet die Möglichkeit, ein großes Bildungspotential zu entfalten und einen besonderen Zugang zur Lösung praktischer Probleme anzubieten; daneben stellt sie eine gute Voraussetzung für interdisziplinäre Forschung dar.

Unser Fach war während Jahrzehnten auf die Lehrerausbildung ausgerichtet. Seit den 1960er Jahren ist eine Verlagerung der Haupttätigkeitsbereiche der Diplomgeographen festzustellen. So hat die Geographie im Rahmen der Raumplanung an praktischer Bedeutung gewonnen. Im Bereiche der Verwaltung und zahlreicher Branchen der Privatwirtschaft sind vermehrt Geographen tätig. Doch ist die Situation in mehrfacher Hinsicht unbefriedigend: Das Image des Berufsgeographen bleibt unscharf, so da $ß$ sich die Stellensuche junger Geographen des öfteren schwierig gestaltet und die Bedeutung geographischer Standpunkte bei zahlreichen wichtigen Problemen nicht genügend Beachtung findet. Das vorliegende «Leitbild Geographie Schweiz» soll zur Verbesserung dieser Situation Orientierungshilfen für Lehre, Forschung, Praxis und Schule bieten.

Angeregt durch die Schweizerische Gesellschaft für angewandte Geographie (SGAG), haben die Schweizerische Geographische Gesellschaft (SGgG) und die Schweizerische Geographische Kommission $(\mathrm{SGgK})$ in den letzten zwei Jahren mehrere Arbeitsgruppen eingesetzt, welche die verschiedenen Aspekte der aktuellen Problematik ausgeleuchtet haben. Die Ergebnisse wurden an zwei Geographentagen (2./3. Mai 1986 in Bern und 8./ 9. Mai 1987 in Fribourg) vorgestellt und diskutiert. Das vorliegende Leitbild stellt eine Zusammenfassung dieser Aussprachen und Beschlüsse dar. Seine Verwirklichung ist für die Zukunft unseres Faches eine unabdingbare Notwendigkeit. Es handelt sich darum, die seit Jahren in der SGgK erreichte Kooperation zwischen Geographischen Instituten und Fachgesellschaften zu vertiefen und die problemlösungsorientierte Seite des Faches vermehrt in den Vordergrund zu stellen.

Die Arbeitsgruppe, welche sich mit der Ausarbeitung des Leitbildes befaßte, bestand aus dem Redaktor sowie den Herren Prof. Dr. K. Aerni, Bern; Prof. Dr. G. Furrer, Zürich; Prof. Dr. D. Steiner, Zürich; Dr. B. Werlen, Zürich und Prof. Dr. J. Winistörfer, Lausanne. Die endgültige Fassung wurde am 5. Dezember 1987 abgeschlossen.

\section{Zur Zielsetzung der Geographie}

Die Geographie befindet sich an der Schnittstelle von Natur-, Sozial- und Geisteswissenschaften. Sie untersucht den Erdraum (die Landschaft) im Hinblick auf seine Differenzierung, Organisation und Umwandlung. Dabei interessiert sie sich vor allem für die Erfassung der Bedeutung der erdräumlichen Dimension sowohl im Leben jedes Einzelnen als auch im menschlichen Zusammenleben. Somit befaßt sich die Geographie mit dem Interaktionsdreieck der Systeme Landschaft, Mensch (als Individuum) und Gesellschaft, und zwar wie folgt: Sie betrachtet die Wechselwirkungen sowohl innerhalb als

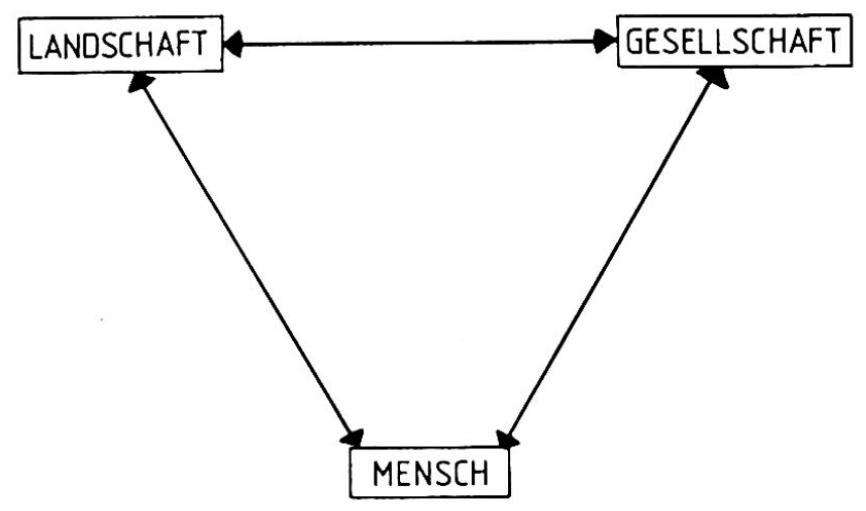

Walter Leimgruber, Prof. Dr., Präsident der Schweiz. Geogr. Gesellschaft, Institut de Géographie, Université de Fribourg, Pérolles, 1700 Fribourg 
auch zwischen den Systemen, insofern sie räumliche Ursachen und Wirkungen in der Landschaft haben.

Damit wird der Landschaftsbegriff der klassischen Geographie mit einer modernen (human)ökologischen Perspektive verbunden, die den Menschen als handelndes Lebewesen mit einer Umwelt und einer Mitwelt sieht, die er beide beeinflußt, von denen er aber auch beeinflußt wird.

Kurz: die Geographie beschäftigt sich mit der Entwicklung der Landschaft zum heutigen Zustand hin. Dabei untersucht sie die Folgen menschlicher Handlungen für die Landschaft und deren Rückwirkungen auf menschliche Tätigkeiten. Damit erarbeitet sie Grundlagen zur Beurteilung der künftigen Entwicklung, der Erhaltung oder der Regenerierung eines vielfältig genutzten Lebensraumes, den sie über praxisorientierte Arbeitsrichtungen mitgestaltet. Diese Grundlagen ermöglichen ihr, bei der Gestaltung der künftigen Raumentwicklung mitzuwirken. Damit trägt die Geographie zur Lösung räumlicher Probleme bei.

\section{Geographie an der Hochschule}

\subsection{Leitlinien für die Lehre}

Die Dozenten sollen bei ihrer Arbeit didaktische Gesichtspunkte berücksichtigen und wissenschaftliche Ergebnisse methodisch aufgearbeitet präsentieren.

Der Umsetzung von Forschungsergebnissen durch die Zusammenarbeit von Instituten und Berufsverbänden (VSGg, SGAG) ist volle Aufmerksamkeit zu schenken. Dies setzt einen breiten Forschungsbegriff voraus: auch die didaktische Aufbereitung von Forschungsergebnissen soll Bestandteil der Forschungsarbeit bilden. Zudem soll die Ausbildung vermehrt die wichtigsten Tätigkeitsbereiche des $\mathrm{Be}$ rufsgeographen berücksichtigen.

Das Grundstudium muß ein breites geographisches Grundwissen in den klassischen Teildisziplinen der Physischen Geographie und der Humangeographie enthalten (theoretische und begriffliche Grundlagen, elementare Modelle, praktische Arbeitsmethoden) sowie Grundkenntnisse in der Kartographie und geographischen Datenverarbeitung (inkl. EDV) vermitteln.

Das Hauptstudium soll fundierte theoretische und methodische Kenntnisse in ein bis zwei Teildisziplinen mit aktuellen Forschungsarbeiten des betreffenden Instituts kombinieren und damit die Fähigkeit schulen, Probleme zu erkennen und zu lösen. Auf der Stufe des Hauptstudiums unterscheiden sich die einzelnen Institute auf Grund ihrer Lehrund Forschungsschwerpunkte. Ein individuelles Berufspraktikum und die Pflege von Fremdspra- chen werden jedem Studenten und jeder Studentin empfohlen.

In Grund- und Hauptstudium sind Veranstaltungen vorzusehen, welche die geographischen Fragestellungen im gesamten behandeln und die Teilaspekte thematisch und methodisch verbinden. So ist auf allen Stufen der Ausbildung der Sinn für räumliche Zusammenhänge durch Exkursionen und Geländepraktika zu schulen.

Das Studium der Nebenfächer soll ermöglichen, aus relevanten Nachbardisziplinen Transferwissen zu erwerben und adäquat auf geographische Problemstellungen anzuwenden. Durch die gegenseitige Anerkennung der Vordiplome ist der Übertritt an andere Hochschulen nach Abschluß des Grundstudiums zu erleichtern.

\subsection{Leitlinien für die Ausbildungsinhalte}

Um die genannten Ziele zu erreichen, sollten der Geograph/die Geographin über Kompetenz in folgenden Ausbildungsinhalten verfügen:

\section{Nach dem Grundstudium:}

Kenntnis der Fachsprache, der Basisliteratur, der Fragestellungen der Geographie und der Gegenwartsprobleme. Beherrschen der wichtigsten Grundtechniken der Geographie (empirische Forschungstechniken, Geländeaufnahmen, Grafik, Fernerkundung, Kartographie, Statistik, Datenverarbeitung/EDV, Bibliographie, Medientechnik), Fähigkeit, sich mündlich und schriftlich auszudrükken in Diskussionen, Vorträgen und schriftlichen Arbeiten.

Folgende Themenbereiche sollten in dieser Phase vermittelt werden:

- Allgemeine physische Geographie, Klimatologie, Geomorphologie, Biogeographie, unter Umständen Hydrologie und Pedologie

- Allgemeine Humangeographie, Kultur- und Sozialgeographie, Bevölkerungsgeographie, Wirtschaftsgeographie, unter Umständen Siedlungsund Verkehrsgeographie

- Einführung in Raumplanung und Umweltfragen

- Grundlagen der verschiedenen Arbeitsmethoden

\section{Beim Diplomabschluß:}

a) Überblick über die verschiedenen geographischen Fragestellungen, Einführung in die Wissenschaftstheorie für Geographen.

b) Theoretisch-methodische Spezialisierung in ein bis zwei geographischen Teildisziplinen mit zugehörigen Beobachtungs-, Meß-, Befragungs-, Bild- und Datenverarbeitungstechniken.

c) Fähigkeit zur wissenschaftlichen Einarbeitung in raumbezogene Probleme. Insbesondere sollten an den geographischen Instituten der schweizerischen Hochschulen Studiengänge eingeführt 
werden, die explizit und praxisorientiert auf die Tätigkeitsbereiche Raumplanung (Siedlungs-, Verkehrs- und Regionalplanung), Regionalforschung und -entwicklung (Regionalpolitik, Dritte Welt), Landschaftsgestaltung (Landschaftsverträglichkeit) und Umweltfragen ausgerichtet sind.

d) Breites geographisches Grundwissen und Kommunikationsfähigkeit mit Nachbardisziplinen (= Voraussetzungen zur interdisziplinären Zusammenarbeit). Kenntnis der allgemeinen Theorien und Methoden mindestens einer Nachbardisziplin im Hinblick auf eine Anwendung in geographischen Fragestellungen. Fähigkeit zum selbständigen Abfassen von Seminar- und Lizenziats- bzw. Diplomarbeit.

e) Im Hinblick auf die spätere Berufstätigkeit ist eine praxisorientierte berufsvorbereitende Veranstaltung (Seminar, Praktikum) einzuführen (Vorschläge des Geographentages 1987 und der SGAG).

\subsection{Schwerpunkte in der Forschung}

Die Hauptaufgaben der Institute bilden Lehre und Forschung. Diese beiden Arbeitsbereiche sind an den Hochschulen nebeneinander und komplementär zu pflegen. Ergebnisse der Forschung haben in die Lehre einzufließen. Die Forschung bestimmt somit zum Teil den Inhalt der Vorlesungen, Übungen und Seminarien. Dabei werden Forschungsergebnisse in erster Linie im Hauptstudium vermittelt.

Die geographische Forschung in der Schweiz orientiert sich an den Gegenwartsproblemen im MenschUmwelt-System. Damit leistet sie nicht nur einen aktiven Beitrag zur Lösung dieser Probleme - die sowohl auf der ökologischen wie auch auf der sozialen Ebene angesiedelt sind -, sondern sie führt sie über die Lehre auch in die Ausbildung der Geographielehrer ein, denen bei der Weitervermittlung geographischen Gedankenguts eine entscheidende Rolle zukommt (s. Kap. 5). Die Institute sind letztlich der Ort, wo sich Forschung, Vermittlung und Erfahrungen aus der Praxis überschneiden.

Es ist nicht Aufgabe dieses Leitbildes, den Instituten ihre Forschungsinhalte vorzuschreiben. Hingegen soll eine minimale Koordination im Rahmen der «Hochschule Schweiz» angestrebt werden, damit Doppelspurigkeiten unterbleiben. Die Institute bezeichnen daher ihre Forschungsschwerpunkte (thematisch und räumlich). Damit wird den in der SGgK zusammengeschlossenen Instituten und Fachgesellschaften eine Übersicht zur Verfügung stehen, die es erlaubt

a) den Forschungsprozess transparent zu gestalten,

b) Forschungszusammenarbeit zu ermöglichen,

c) neue Projekte anzuregen,

d) Forschungslücken aufzuzeigen.

\section{Praxisorientierte Ausbildung - Berufsgeograph}

Den in der Praxis tätigen Geographen muß der Kontakt zur Hochschule im Sinne der Weiterbildung, der Nachwuchsförderung und der fachlichen sowie methodischen Unterstützung besser gewährleistet werden als bisher. Umgekehrt erwartet auch die Hochschule von den Praktikern eine Rückkoppelung in diesen Bereichen. Zur Vertiefung der gegenseitigen Beziehungen ist vorzusehen:

a) Ausbildung an der Hochschule

Methodische und materielle Kompeten $z$ wird durch inhaltliche Anpassung der Studienpläne verbessert. Mit Blick auf den Arbeitsmarkt müssen dazu in den nächsten Jahren namentlich die Bereiche Raumplanung (insbesondere Wohnlichkeit und Entwicklung der Siedlungen), Umweltfragen (Mensch-UmweltBeziehung), Regionalpolitik (integrale Ansätze) sowie Entwicklungsgebiete vertieft und praxisorientiert ausgebildet werden.

Organisatorische Kompetenz wird von den Berufsverbänden (SGAG, VSGg) vermittelt.

Anwendungskompetenz soll im Unterricht verbessert werden (Berücksichtigung fachexterner Themen - Nebenfächer - und ihre Anwendung auf geographische Probleme, schärfere Beurteilung und Schulung der Teilnehmer bei ihren Vorträgen und in den Diskussionen).

b) Praktiker an der Hochschule

An den meisten geographischen Instituten der Schweiz lehren auch Praktiker, u. a. Vertreter der Raumplanung. Ein Ausbau dieser Lehraufträge ist anzustreben.

\section{c) Berufspraktikum}

Im Hinblick auf das individuelle Praktikum sind die Berufsgeographen aufgerufen, vermehrt Praktikanten aufzunehmen.

d) Weiterbildung und Orientierung

Sowohl an der Hochschule als auch in Schule und Praxis ist eine dauernde Weiterbildung nötig. In diesem Sinne ist, wie das bisher bereits für die Geographielehrer der Fall war, auch den angewandt arbeitenden Geographen eine Weiterbildung durch die Institute anzubieten (in Zusammenarbeit mit der SGAG). Der Informationsfluß zwischen Hochschule und Praxis läßt sich durch spezielle Seminarien verbessern.

\section{Geographie in den Beziehungen Hochschule - Schule - Erwachsenenbildung}

Die aktuellen Raum- und Umweltprobleme erfordern die Bearbeitung von komplexen Themen im Geographieunterricht. Die fachdidaktische Ausbildung ist deshalb diesen Erfordernissen anzupassen. 
a) Durch Aufbau und Ausbau der fachdidaktischen Abteilungen an den Universitäten soll der Fachdidaktiker vom Schuldienst entlastet werden. $\mathrm{Zu}$ seinem Tätigkeitsbereich gehören:

- Mitwirkung bei der Gestaltung der Studienpläne

- Mitarbeit bei Fortbildungsveranstaltungen

- Umsetzung von Forschungsergebnissen

b) Ein besonderes Gewicht soll die Ausbildung im Umgang mit Medien erhalten, wobei der Fachdidaktiker durch eine Ausweitung seines Auftrages auch in den allgemeinen Unterricht der Institute einbezogen werden kann.

c) Die Bildung einer ständigen Konferenz der Fachdidaktiker ist anzustreben.

d) Der Verein Schweizerischer Geographielehrer (VSGg) bietet Kurse an, bei denen er seinen Mitgliedern Forschungsergebnisse und Erfahrungen aus der Praxis vermittelt.

e) Zur Förderung der Erwachsenenbildung ist die Zielsetzung des fachdidaktischen Unterrichts zu öffnen und auf die Öffentlichkeitsarbeit auszudehnen.

f) Es soll darauf hingearbeitet werden, daß der Geographieunterricht an den Mittelschulen verstärkt berücksichtigt wird. Die Lehrpläne sind den neuen Erfordernissen unserer Gesellschaft anzupassen, und der Unterricht ist bis in die Maturklassen zu führen.

\section{Wissenschaftspolitik und Öffentlichkeitsarbeit}

Als raumorientierte Wissenschaft arbeitet die Geographie auf mehreren Maßstabsebenen. So ist die lokale Fallstudie ebenso von Bedeutung wie großräumige und internationale Forschungsprojekte Diese Besonderheit der Geographie muß immer wieder hervorgehoben werden: auch kleinräumige Arbeiten können wesentliche methodische und praktische Beiträge zur Lösung räumlicher Probleme unserer Zeit liefern.

Forschungsinhalte, Aufgabe und Wesen der Geographie sind einer breiten Öffentlichkeit nicht bekannt. Diese Situation ist zu verbessern durch stärkere Öffentlichkeitsarbeit, insbesondere

- vermehrte Medienpräsenz

- Zusammenarbeit (für die Öffentlichkeitsarbeit) zwischen Instituten, Berufsverbänden und Regionalgesellschaften

- entsprechende Ausweitung und Aktualisierung der Tätigkeit der Regionalgesellschaften

- Übernahme mediendidaktischer Elemente in die Ausbildung

- Reorganisation der Schweizer Geographie mit dem Ziel eines größeren Handlungsspielraumes

Zur Verbesserung der Öffentlichkeitsarbeit und der Zusammenarbeit zwischen Instituten, Berufsverbänden, Fachgesellschaften und Regionalgesellschaften soll als neues Organ der SGgG ein Zentralsekretariat geschaffen werden.

Die Geographie ist bereits in mehreren Politikbereichen tätig. Diese Aktivität wird ausführlich im Bericht «Geographie im Jahre 2000» zu Handen der SNG dargestellt. 